\title{
INITIAL VALUE PROBLEMS IN ELASTICITY
}

\author{
ROLF LEIS \\ Institut für Angewandte Mathematik, Universität Bonn \\ Wegelerstr. 10, 5300 Bonn, Germany
}

Introduction. Initial and initial-boundary value problems play an important role in mathematical physics. For instance one can write the wave equation, the plate equation or the system of linear elasticity in the form

$$
u_{t t}+A u=0 \quad \text { with } u(0)=u^{0} \text { and } u_{t}(0)=u^{1} .
$$

Here $A$ is a linear differential operator in a domain $G \subset \mathbb{R}^{3}$. Next,

$$
u_{t}+\mathrm{i} A u=0 \quad \text { with } u(0)=u^{0}
$$

represents the Schrödinger equation, the system of Maxwell's equations or the system of linear acoustics, and by

$$
u_{t}+A u=0 \quad \text { with } u(0)=u^{0}
$$

heat conduction phenomena like initial-boundary value problems in linear thermoelasticity are described. Except for the last example $A$ is a selfadjoint operator.

To solve such problems one has to give a solution concept first. Afterwards one tries to get more specific knowledge of the solutions obtained. For instance one inquires about their regularity, or about their asymptotic behaviour for large times and proves the existence of wave and scattering operators. Problems of inverse scattering theory are of great mathematical and practical interest (data like initial values, boundary or medium shall be recovered from the reflected signals). To treat such problems one has to know, for instance, how the scattering operator is determined by the boundary and to invert this mapping. In the high frequency limiting case one obtains relatively simple formulae. In the following, however, I shall not touch on such questions. Rather, in the first part of the lecture I shall only briefly treat linear boundary value problems for exterior domains, and also with the restriction that the underlying medium is homogeneous outside a sufficiently large ball. Of course boundary value problems for domains with unbounded boundaries are also of great importance. 
Meanwhile linear problems are fairly well understood whereas many nonlinear problems are still open. So the second part of the lecture will be concerned with nonlinear problems, primarily with the existence of global small smooth solutions. Much progress has been made in this field in recent years. So I shall first report on some results given by F. John, S. Klainerman and others and afterwards treat elastic media with cubic symmetry in $\mathbb{R}^{2}$.

1. Linear problems. In the linear case the underlying theory is known and in principle well understood now. Let me shortly report on the development guided by a simple wave equation. The underlying operator is selfadjoint in this case so that the spectral theorem is at our disposal. Similar results can be obtained for other equations of mathematical physics.

So let $G$ be a domain in $\mathbb{R}^{3}$, and let $a_{i k}=a_{k i}(i, k=1,2,3)$ be real-valued, bounded $\mathcal{C}_{1}(G)$-functions with

$$
\exists p_{0}>0 \forall x \in G \forall \xi \in \mathbb{R}^{3} \quad \xi_{i} a_{i k} \xi_{k} \geq p_{0}|\xi|^{2} .
$$

Let the medium be homogeneous and isotropic sufficiently far outside in case of an exterior domain; thus a number $r_{a}>0$ exists with

$$
\forall x \in G_{a}:=\left\{x \in G|| x \mid>r_{a}\right\} \quad a_{i k}=\delta_{i k} .
$$

Since we want to confine ourselves to the Dirichlet boundary value problem let $A: \mathcal{D}(A) \subset \mathcal{L}^{2}(G) \rightarrow \mathcal{L}^{2}(G)$ with

$$
\mathcal{D}(A):=\left\{u \in \mathcal{H}_{1}(G) \mid \partial_{i} a_{i k} \partial_{k} u \in \mathcal{L}^{2}(G)\right\} \quad \text { and } \quad A u:=-\partial_{i} a_{i k} \partial_{k} u .
$$

Then we are looking for a $u: \mathbb{R}_{0}^{+} \times G \rightarrow \mathbb{R}$ with

$$
u_{t t}+A u=0, \quad u(0)=u^{0} \text { and } u_{t}(0)=u^{1} .
$$

Several solution concepts exist for this initial value problem (e.g. there are distribution, weak, or classical solutions). In the following we shall choose the concept of solutions with finite energy which is suggested by physics, namely

$$
E(t):=\left\|u_{t}(t)\right\|^{2}+\left\|A^{1 / 2} u(t)\right\|^{2}<\infty,
$$

that is, with finite sum of kinetic and potential energy $\left(A^{1 / 2}\right.$ corresponds to $\left.\nabla\right)$. For that purpose we have to assume $u_{0} \in \mathcal{D}\left(A^{1 / 2}\right)$ of course; $\|\cdot\|$ denotes the $\mathcal{L}^{2}(G)$-norm.

In the following let $\mathcal{H}:=\mathcal{L}^{2}(G)$, let $\stackrel{\circ}{\mathcal{C}}(G)$ be the set of continuous functions with compact support in $G$, and $\mathcal{V}:=\mathcal{C}(\mathbb{R}, \mathcal{D}(A)) \cap \mathcal{C}_{2}(\mathbb{R}, \mathcal{H})$. Furthermore, let $u^{0}, u^{1} \in \mathcal{H}$. Then $u \in \mathcal{C}\left(\mathbb{R}_{0}^{+}, \mathcal{H}\right)$ is called a weak solution of the wave equation if

$$
\forall \varphi \in \mathcal{V} \quad 0=\int_{\mathbb{R}^{+} \times G} u \overline{\left(\varphi_{t t}+A \varphi\right)}+\left(u^{0}, \varphi_{t}(0, \cdot)\right)-\left(u^{1}, \varphi(0, \cdot)\right) .
$$

This definition goes back to M. Vishik and O. A. Ladyzhenskaya [1956]. If $u^{0} \in$ $\mathcal{D}\left(A^{1 / 2}\right)$ then $u$ is called a solution with finite energy when $u \in \mathcal{C}\left(\mathbb{R}_{0}^{+}, \mathcal{D}\left(A^{1 / 2}\right)\right) \cap$ $\mathcal{C}_{1}\left(\mathbb{R}_{0}^{+}, \mathcal{H}\right)$. 
The initial-boundary value problem just defined is uniquely solvable. This follows relatively easily from spectral or semigroup theory. In our case $A$ is selfadjoint and by

$$
\begin{aligned}
u(t) & :=\cos \left(A^{1 / 2} t\right) u^{0}+A^{-1 / 2} \sin \left(A^{1 / 2} t\right) u^{1} \\
& =\int_{0}^{\infty}\left\{\cos (\sqrt{\lambda} t) d P(\lambda) u^{0}+\frac{\sin (\sqrt{\lambda} t)}{\sqrt{\lambda}} d P(\lambda) u^{1}\right\}
\end{aligned}
$$

we can obtain a solution of the problem. The energy is constant.

Thus we can prove existence and uniqueness of a solution. For special equations many questions, however, remain unanswered, for instance the question of regularity of the solution of Maxwell's equations in the neighbourhood of edges and corners. If one wants to know further particulars about the solution one needs better knowledge of the spectrum of $A$. For $G$ bounded the expansion of $A$ with respect to eigenfunctions and the expansion of $u$ with respect to standing waves follow using spectral theory for compact operators. Namely from Rellich's selection theorem and Poincaré's estimate one easily obtains $A^{-1} \in \mathcal{K}(\mathcal{H})$. But we shall leave it at that.

Rather, in the following we want to be concerned with questions about the time-asymptotic behaviour of the solutions and with scattering problems in unbounded domains. We choose a simple case and assume $G$ to be an exterior domain. So $G$ is the complement of a bounded domain and has bounded boundary.

To describe the asymptotic behaviour of the solutions in exterior domains for large $t$ one starts by treating the "free space" problem, i.e. the case $G=\mathbb{R}^{3}$ with $a_{i k}=\delta_{i k}$. This case can explicitly be gone through using Fourier transformation and thus it is possible to get the asymptotic behaviour of its solutions. It serves for comparison with the solutions of more general exterior boundary value problems afterwards.

To simplify the representation we use complex notation. Let $A_{0}$ be the underlying operator in the free space case and $u_{0}^{0}, u_{0}^{1}$ the real initial values with $u_{0}^{1} \in \mathcal{D}\left(A_{0}^{-1 / 2}\right)$. Let $v_{0}^{0}:=u_{0}^{0}+\mathrm{i} A_{0}^{-1 / 2} u_{0}^{1}$ and $v_{0}(t):=\exp \left(-\mathrm{i} A_{0}^{-1 / 2} t\right) v_{0}^{0}$. Then $u_{0}(t):=\operatorname{Re} v_{0}(t)$ is the solution of the problem. $v_{0}$ is called the free solution. The existence of incoming and outgoing waves

$$
v_{0}^{ \pm}(t, x)=G^{ \pm}\left(|x| \pm t, x_{0}\right) /|x|
$$

with

$$
\lim _{t \rightarrow \infty}\left\|v_{0}(t, \cdot)-v_{0}^{ \pm}(t, \cdot)\right\|=\lim _{t \rightarrow \infty}\left\|v_{0}^{\mp}(t, \cdot)\right\|=0
$$

follows.

To get on in case of more general exterior boundary value problems we have to know more about the spectrum of $A$. We have

$$
\sigma(A)=\sigma_{c}(A)=\sigma_{a c}(A)=\mathbb{R}_{0}^{+} .
$$


To prove this, three results are used. First, from Poincaré's estimate

$$
\left\|\frac{\varphi}{|\cdot|}\right\| \leq 2\|\nabla \varphi\| \quad \text { for all } \varphi \in \stackrel{\circ}{\mathcal{C}}_{1}(G)
$$

$\mathcal{N}(A)=0$ follows, and Rellich's estimate [1943]

$$
\exists p>0 \exists r_{1}>r_{a} \forall r>r_{1} \quad \int_{r_{a}<|x|<r}|u(x)|^{2} d x \geq p r
$$

implies $\sigma_{p}(A)=\emptyset$ for $a_{i k}=\delta_{i k}$. For variable coefficients $\sigma_{p}(A)=\emptyset$ then follows from C. Müller's [1954] principle of unique continuation of the solutions of elliptic equations.

Next one again looks at the time harmonic case (expansion of solutions with respect to standing waves) and solves the Dirichlet problem for the Helmholtz equation $\partial_{i} a_{i k} \partial_{k} u+\lambda u=0$ for $\lambda \in \mathbb{R}^{+}$. Since $\lambda$ does belong to the spectrum of $A$ this is not a standard boundary value problem. Rather, one speaks of "exterior boundary value problems" and looks for solutions in a space which is larger than $\mathcal{H}$. The asymptotic behaviour for large $|x|$ of such solutions is prescribed then. In fact, one makes an incoming or outgoing Sommerfeld radiation condition $\left(x_{0} \nabla \mp\right.$ $\mathrm{i} \sqrt{\lambda}) u \in \mathcal{L}^{2}(G)$.

For $a_{i k}=\delta_{i k}$ uniqueness of the solution of the exterior Dirichlet problem was shown by V. D. Kupradze [1934], H. Freudenthal [1939] and F. Rellich [1943]. Existence was proved by I. N. Vekua [1943], comp. [1967], H. Weyl [1952], and C. Müller [1952] using the integral equation method. Variable coefficients were dealt with using Hilbert space methods afterwards.

The solvability of exterior boundary value problems has led to the definition of generalized eigenfunctions and to expansions with respect to them. This has been used repeatedly, especially the analogue of the Fourier transform in $\mathbb{R}^{n}$ for exterior domains has been given. This development goes back to T. Ikebe [1960], compare also C. H. Wilcox [1975].

Finally, let us point out that D. M. Ėŭdus [1962] proved the "principle of limiting absorption". This means that for $f \in \mathcal{L}^{2 f}(G)\left(\mathcal{L}^{2}\right.$-functions with finite support) one obtains the outgoing or incoming solution $u^{ \pm}$of $-\partial_{i} a_{i k} \partial_{k} u-\lambda u=f$ by taking the limit

$$
u=\lim _{\varepsilon \downarrow 0}\left(A-(\lambda \pm \mathrm{i} \varepsilon)^{-1}\right) f .
$$

This limit exists in a weighted $\mathcal{H}_{1}(G)$-norm. Since $\mathcal{L}^{2 f}(G)$ is dense in $\mathcal{H}$, it follows from Stone's formula that the spectrum of $A$ is absolutely continuous and thus, since $\sigma_{p}(A)=\emptyset$ [comp. T. Kato 1976, p. 518],

$$
\mathcal{H}=\mathcal{H}_{a c}:=\{x \in \mathcal{H} \mid(P(\lambda) x, x) \text { is absolutely continuous }\} .
$$

With that we have put together the necessary results from the theory of the Helmholtz equation and we continue treating the wave equation. The "principle of local energy decay" immediately follows from the absolute continuity of the 
spectrum and the Riemann-Lebesgue lemma, so $\forall K \subset G, K \Subset \mathbb{R}^{3}$,

$$
\lim _{t \rightarrow \infty}\left\{\left\|A^{1 / 2} u(t)\right\|_{\mathcal{L}^{2}(K)}+\left\|u_{t}(t)\right\|_{\mathcal{L}^{2}(K)}\right\}=0 .
$$

Therefore one expects that for $t$ sufficiently large $u(t)$ behaves like a free solution. To be more precise, let $J_{G}: \mathcal{H} \rightarrow \mathcal{H}_{0}, \mathcal{H}_{0}:=\mathcal{L}^{2}\left(\mathbb{R}^{3}\right)$, with $\left(J_{G} u\right)(x):=$ $u(x)$ for $x \in G$, null otherwise. Then one looks for $v_{0}^{+}$and $v_{0}^{-} \in \mathcal{H}_{0}$ with

$$
\lim _{t \rightarrow \pm \infty}\left\|J_{G} \mathrm{e}^{-\mathrm{i} A^{1 / 2} t} v^{0}-\mathrm{e}^{-\mathrm{i} A_{0}^{1 / 2} t} v_{0}^{ \pm}\right\|=\lim _{t \rightarrow \pm \infty}\left\|\mathrm{e}^{\mathrm{i} A_{0}^{1 / 2} t} J_{G} \mathrm{e}^{-\mathrm{i} A^{1 / 2} t} v^{0}-v_{0}^{ \pm}\right\|=0 .
$$

From the absolute continuity of the spectrum of $A$ the existence of unitary wave operators $W^{ \pm}: \mathcal{H} \rightarrow \mathcal{H}_{0}$ with

$$
W^{ \pm}:=\operatorname{s-lim}_{t \rightarrow \pm \infty} \mathrm{e}^{\mathrm{i} A_{0}^{1 / 2} t} J_{G} \mathrm{e}^{-\mathrm{i} A^{1 / 2} t}
$$

follows. Therefore $v_{0}^{ \pm}:=W^{ \pm} v^{0}$ exist. Then $S:=W^{+}\left(W^{-}\right)^{*}$ is the scattering operator and we have $v_{0}^{+}=S v_{0}^{-}$.

There are different methods to prove the existence of wave operators. P. Lax and R. S. Phillips [1967] use semigroup theory, C. H. Wilcox [1975] the expansion with respect to generalized eigenfunctions, and T. Kato [comp. 1976], A. L. Belopol'skı̌ and M. Sh. Birman [1968], and D. B. Pearson [1978] as well make use of results from perturbation theory. For that purpose one has to show that the general case can be interpreted as a nuclear perturbation of the free case. More results from scattering theory and especially from quantum scattering theory can be found in the four-volume book of M. Reed and B. Simon [1972-1979].

So far we have indicated how solutions of initial-boundary value problems can be approximated by free space solutions for large times. As was already said there are other limiting cases where approximation by simpler solutions is possible. The limiting case of high frequencies belongs to this, as does the limit to geometrical optics. These investigations are important in inverse scattering theory.

Dealing with nonlinear problems it became important during the last years to prove good decay estimates for the solutions of linear equations and large time. So-called $\mathcal{L}^{p}$ - $\mathcal{L}^{q}$ estimates have been proved. By that we mean estimates of the form

$$
\|D u(t)\|_{\mathcal{L}^{q}} \leq c_{q}(1+t)^{-1+2 / q}\left\|u^{0}\right\|_{\mathcal{L}_{, N_{p}}^{p}}, \quad D:=\left(\partial_{t}, \nabla\right),
$$

with $q \geq 2,1 / p+1 / q=1,1 \leq p \leq 2$ and $0<N_{p}$. In the next chapter we shall go into this more closely.

\section{Nonlinear problems}

2.1. Conservation laws. A typical example in $\mathbb{R}^{1}$ is given by

$$
u_{t}+f(u)_{x}=0 .
$$

Choosing

$$
f(u)=\frac{1}{2} u^{2}
$$


we obtain the Burgers equation. The traffic equation, also, is of this type. In $\mathbb{R}^{1}$ one can obtain solutions by solving their characteristic equations. It is important that, generally speaking, one encounters shock waves. The Rankine-Hugoniot condition together with an entropy condition provide, however, unique solvability in the weak sense. We do not intend to go into details here but again immediately switch to the wave equation.

2.2. Wave equations. To start with let us think of the vibrating string or vibrating membrane. An example is provided by

$$
y_{t t}-\left(\frac{y_{x}}{\sqrt{1+y_{x}^{2}}}\right)_{x}=0 .
$$

With $g(x):=\sqrt{1+x^{2}}-1=x^{2} / 2-x^{4} / 8+\ldots$,

$$
u:=D y=\left(\begin{array}{c}
y_{t} \\
y_{x}
\end{array}\right) \quad \text { and } \quad A:=-\left(\begin{array}{cc}
0 & \text { div } \\
\operatorname{grad} & 0
\end{array}\right)
$$

we get

$$
\square y=-y_{x x}+g^{\prime}\left(y_{x}\right)_{x}=-\frac{3}{2}\left(y_{x}\right)^{2} y_{x x}+\ldots=: f(u, \nabla u)
$$

or

$$
u_{t}-\left(\begin{array}{c}
g^{\prime}\left(u_{2}\right) \\
u_{1}
\end{array}\right)_{x}=0
$$

respectively

$$
u_{t}+A u=\left(\begin{array}{c}
f(u, \nabla u) \\
0
\end{array}\right)=: F(u, \nabla u)
$$

with $|F(u, \nabla u)| \leq c(|u|+|\nabla u|)^{3}$ for small $u$. The energy

$$
E(t)=\int\left(\frac{1}{2} u_{1}^{2}+g\left(u_{2}\right)\right)(t)
$$

is conserved in this case.

In $\mathbb{R}^{1}$ solutions of nonlinear wave equations always develop singularities as was shown by F. John [1974] and [1976]. For large data the same generally holds in $\mathbb{R}^{n}$. The "life-span" of such solutions was defined and estimated by F. John [1981]. On the other hand, already K. Jörgens [1961] proved global existence for large data, $\square y=f(y)$, and special $f=f(y)$ with cubic growth in $\mathbb{R}^{3}$, and another example even for an $f$ with quadratic growth but small data was given by L. Nirenberg, namely

$$
\square y=y_{t}^{2}-(\nabla y)^{2},
$$

with $y(0)=0$ and $y^{\prime}(0)=g$. On setting $z:=\mathrm{e}^{y}$ this reads

$$
\square z=0, \quad z(0)=1, \quad z^{\prime}(0)=g .
$$

Choosing $\|\nabla g\|_{\mathcal{L}^{1}}+\|g\|_{\mathcal{L}^{\infty}}$ sufficiently small one obtains $0<z(t)<\infty$ from Kirchhoff's representation formula, and $y(t)$ thus exists for all $t$.

S. Klainerman [1980] and [1982] proved global existence of smooth solutions for a large class of right-hand sides and small data. He used a quite complicated 
iteration scheme together with the Nash-Moser technique (comp. J. Moser [1961] and L. Hörmander [1976]).

In a joint paper with G. Ponce [1983] he then first uses a local existence theorem going back to J. Schauder [1935] and T. Kato [1975a, b]. As was already mentioned such solutions will explode after some time and their life-span depends on the size of the initial data. Next they procure good decay estimates of the solutions of the linearized equation.

The idea then is to choose the initial data so small that the life-span is long enough until the linear influence of spreading dominates and prevents the solution from exploding.

Thus one can avoid the iteration scheme and give an elegant existence proof. Let us indicate the essential steps in $\mathbb{R}^{3}$. We assume

$$
|F(u, \nabla u)| \leq c(|u|+|\nabla u|)^{3}
$$

for small $|u|+|\nabla u|$.

Let $v(t)=\mathrm{e}^{-A t} v^{0}$ be the solution of the linear equation. From energy conservation and Kirchhoff's formula it follows that

$$
\|v(t)\|_{\mathcal{L}^{2}}=\left\|v^{0}\right\|_{\mathcal{L}^{2}} \quad \text { and } \quad\|v(t)\|_{\mathcal{L}^{\infty}} \leq c_{\infty}(1+t)^{-1}\left\|v^{0}\right\|_{\mathcal{L}_{, 3}^{1}} .
$$

Derivatives up to the third order appear on the right-hand side. Using interpolation methods, or a result by Pecher [1976], an " $\mathcal{L}^{p}-\mathcal{L}^{q}$ estimate" follows, namely

$$
\|v(t)\|_{\mathcal{L}^{q}} \leq c_{q}(1+t)^{-1+2 / q}\left\|v^{0}\right\|_{\mathcal{L}_{, N_{p}}^{p}}
$$

with $q \geq 2,1 / p+1 / q=1,1 \leq p \leq 2$ and $3(2-p) / p \leq N_{p}<3(2-p) / p+1$.

Let us briefly indicate a proof of the $\mathcal{L}^{\infty}$-estimate. Simplifying we choose

$$
y(0)=0 \quad \text { and } \quad y_{t}(0)=g .
$$

From Kirchhoff's formula we then know

$$
y(t, x)=\frac{t}{4 \pi} \int_{S^{2}} g(x+t \omega) d \omega=: \frac{t}{4 \pi} Q(t),
$$

where $v=D y$. We want to estimate $Q$. This will be done in two steps.

$t$ large: For $t \geq 1$ we have

$$
Q(t)=\int_{S^{2}} \omega \cdot \omega g(\cdot+t \omega) d \omega=\frac{1}{t^{3}} \int_{|y|<t} \operatorname{div}\{y g(\cdot+y)\} d y
$$

and therefore

$$
\|Q(t)\|_{\mathcal{L}^{\infty}} \leq \frac{1}{t^{2}}\|g\|_{\mathcal{L}_{, 1}^{1}}
$$

$t$ small: For $t \leq 1$ we have

$$
|Q(t)| \leq \int_{S^{2}}|g(\cdot+t \omega)| d \omega .
$$


Fubini's theorem yields

$$
\|Q(t)\|_{\mathcal{L}^{1}} \leq 4 \pi\|g\|_{\mathcal{L}^{1}}
$$

and also the corresponding estimates of the derivatives. Since $\mathcal{L}_{, 3}^{1} \hookrightarrow \mathcal{L}^{\infty}$ we get

$$
\|Q(t)\|_{\mathcal{L}^{\infty}} \leq c\|g\|_{\mathcal{L}_{, 3}^{1}} .
$$

The local existence theorem is used next to obtain a solution

$$
u \in \mathcal{C}\left([0, T], \mathcal{H}_{s}\right) \cap \mathcal{C}_{1}\left([0, T], \mathcal{H}_{s-1}\right)
$$

of our equation in $[0, T]$ with $T>1$. We get

$$
\forall t \in[0, T] \quad\|u(t)\|_{\mathcal{L}^{\infty}}+\|\nabla u(t)\|_{\mathcal{L}^{\infty}}<1
$$

assuming $\left\|u^{0}\right\|_{s}$ to be small. $\left(\|\cdot\|_{s}\right.$ denotes the $\mathcal{L}^{2}$-norm of all derivatives up to order $s, s \geq 3$.)

"Energy estimates" are then proved in the third step by elementary but tricky partial integration and by using Gronwall's lemma, namely

$$
\|u(t)\|_{s} \leq c_{s}\left\|u^{0}\right\|_{s} \exp \left\{c_{s} \int_{0}^{t}\left(\|u(r)\|_{\mathcal{L}^{\infty}}^{2}+\|D u(r)\|_{\mathcal{L}^{\infty}}^{2}\right) d r\right\} .
$$

To present an example we take the simple case $s=0$ and $F(u, \nabla u)=y_{x}^{2} y_{x x}$. From

$$
\left(y_{t t}-\Delta y, y_{t}\right)=\left(y_{x}^{2} y_{x x}, y_{t}\right)
$$

we get

or

$$
\frac{1}{2} \frac{d}{d t}\left(\left\|y_{t}\right\|^{2}+\|\nabla y\|^{2}\right) \leq\|u\|^{2}\|u\|_{\infty}\|\nabla u\|_{\infty}
$$

$$
\|u\|^{2} \leq\left\|u^{0}\right\|^{2}+2 \int_{0}^{t}\|u\|^{2}\left\{\|u\|_{\infty}^{2}+\|\nabla u\|_{\infty}^{2}\right\}(s) d s .
$$

From Gronwall's lemma the proposition then follows.

The fourth step is essential. Setting $\tau:=3, \varrho:=\tau+N_{6 / 5}=5$ and $\sigma:=1+\varrho=6$ we define

$$
M_{\tau}(T):=\sup _{t \in[0, T]}(1+t)^{2 / 3}\|u(t)\|_{\mathcal{L}_{, \tau}^{6}}
$$

and show that a constant $M_{0}$, independent of $T$, exists such that

$$
M_{\tau}(T) \leq M_{0} \quad \text { for small } u^{0} \in \mathcal{L}_{, \sigma}^{2} \cap \mathcal{L}_{, \varrho}^{6 / 5} .
$$

To prove this estimate one uses inequalities for products of functions

$$
\|F(u, \nabla u)\|_{\mathcal{L}_{, \varrho}^{6 / 5}} \leq c\|u\|_{\mathcal{L}_{, \sigma}^{2}}\|u\|_{\mathcal{L}_{, \tau}^{6}{ }^{6}}^{2},
$$

as well as

$$
\|u\|_{\mathcal{L}^{\infty}}+\|D u\|_{\mathcal{L}^{\infty}} \leq c\|u\|_{\mathcal{L}_{, \tau}^{6}} .
$$


The latter estimate follows from the differential equation and Sobolev's inequality. Thus we obtain

$$
\begin{aligned}
& \|F(u, \nabla u)\|_{\mathcal{L}_{, e}^{6 / 5}} \leq c\|u\|_{\mathcal{L}_{, \sigma}^{2}}\|u\|_{\mathcal{L}_{, \tau}^{6}}^{2} \\
& \leq c\|u\|_{\mathcal{L}_{, \tau}^{6}}^{2} \delta \exp \left\{c \int_{0}^{t}\left(\|u\|_{\mathcal{L}^{\infty}}^{2}+\|D u\|_{\mathcal{L}^{\infty}}^{2}\right)(r) d r\right\} \\
& \leq c\|u\|_{\mathcal{L}_{, \tau}^{6}{ }_{,} \delta}^{2} \delta \exp \left\{c \int_{0}^{t} \frac{M_{\tau}(T)^{2}}{(1+r)^{4 / 3}} d r\right\} \leq c\|u\|_{\mathcal{L}_{, \tau}^{6}}^{2} \delta \exp \left\{c M_{\tau}(T)^{2}\right\} .
\end{aligned}
$$

By the $\mathcal{L}^{p}-\mathcal{L}^{q}$ estimate $(2.3)$ with $q=6$ and $p=6 / 5$, it follows from

$$
u(t)=\mathrm{e}^{-A t} u^{0}+\int_{0}^{t} \mathrm{e}^{-A(t-r)} F(u, \nabla u)(r) d r
$$

that, with $x:=M(T)$,

$$
\begin{aligned}
(1+t)^{2 / 3}\|u(t)\|_{\mathcal{L}_{, \tau}^{6}} & \leq c\left\{\delta+\int_{0}^{t}\left(\frac{1+t}{1+t-r}\right)^{2 / 3}\|F(u, \nabla u)\|_{\mathcal{L}_{, \varrho}^{6 / 5}}(r) d r\right\} \\
& \leq c \delta\left\{1+c x^{2} \mathrm{e}^{c x^{2}} \int_{0}^{t}\left(\frac{1+t}{1+t-r}\right)^{2 / 3}\left(\frac{1}{1+r}\right)^{4 / 3} d r\right\}
\end{aligned}
$$

or

$$
x \leq c \delta\left(1+x^{2} \mathrm{e}^{c x^{2}}\right) \quad \text { with } \delta:=\left\|u^{0}\right\|_{\mathcal{L}_{, \sigma}^{2}}+\left\|u^{0}\right\|_{\mathcal{L}_{, \varrho}^{6 / 5}} .
$$

For $\delta$ sufficiently small this implies $M_{\tau}(T) \leq M_{0}$.

An a priori estimate for the local solution $u(t)$ follows for small $\delta$, namely

$$
\begin{aligned}
\|u(t)\|_{s} & \leq c\left\|u_{0}\right\|_{s} \exp \left\{c \int_{0}^{t}\left(\|u\|_{\mathcal{L}^{\infty}}^{2}+\|D u\|_{\mathcal{L}^{\infty}}^{2}\right)(r) d r\right\} \\
& \leq c\left\|u_{0}\right\|_{s} \exp \left\{c \int_{0}^{t}\|u(r)\|_{\mathcal{L}_{, \tau}^{6}}^{2} d r\right\} \\
& \leq c\left\|u_{0}\right\|_{s} \exp \left\{c M_{\tau}(T)^{2} \int_{0}^{t} \frac{d r}{(1+r)^{4 / 3}}\right\} \\
& \leq K\left\|u_{0}\right\|_{s} \quad \text { with } c=c_{s}, K=K_{s} .
\end{aligned}
$$

Thus

$$
\exists K_{\sigma} \text { independent of } T \forall t \in[0, T] \quad\|u(t)\|_{\sigma} \leq K_{\sigma}\left\|u^{0}\right\|_{\sigma} .
$$

Therefore one can apply the local existence theorem once again and obtain the desired global solution

$$
u \in \mathcal{C}\left([0, \infty), \mathcal{H}_{\sigma}\right) \cap \mathcal{C}_{1}\left([0, \infty), \mathcal{H}_{\sigma-1}\right)
$$

for $u^{0}$ sufficiently small. 
The previous results also show for $t \rightarrow \infty$

$$
\|u(t)\|_{\mathcal{L}_{, \tau}^{6}}+\|u(t)\|_{\mathcal{L}^{\infty}}+\|D u(t)\|_{\mathcal{L}^{\infty}}=\mathcal{O}\left(t^{-2 / 3}\right) .
$$

Corresponding results hold in $\mathbb{R}^{n}$ for $n \geq 2$. For $n \geq 6$ one can allow quadratic growth in eq. (2.1). This also holds for $n=4,5$. The proof and especially estimate $(2.2)$ have to be modified, however, by replacing the $\mathcal{L}^{1}$-norm by the $\mathcal{L}^{2}$ norms of suitable $\Gamma u^{0}$. The $\Gamma$ are differential operators leaving the wave equation invariant. The Lorentz transformation is an example (comp. S. Klainerman [1985], D. Christodoulou [1986] and F. John [1987]).

Even in $\mathbb{R}^{3}$ global smooth solutions exist with quadratic growth in eq. (2.1), if the nonlinearity fulfills a "null condition" [S. Klainerman 1986]. Nirenberg's example has that structure.

The methods of Klainerman and Ponce may be extended to many differential equations of mathematical physics. In particular, problems in elasticity were treated by F. John. Our group did some work on thermoelasticity. These equations are of special interest because of the coupling of a hyperbolic and a parabolic equation. There are always vibrations in $\mathbb{R}^{n}, n \geq 2$. R. Racke [1990c] was able to show that in $\mathbb{R}^{3}$ solutions generally explode; for small data, however, global smooth solutions exist. S. Jiang [1988] solved special initial-boundary value problems in $\mathbb{R}^{1}$. He also proved $\mathcal{L}^{p}-\mathcal{L}^{q}$ estimates for the plate equation with damping. R. Racke [1990d] used the generalized Fourier transformation (generalized eigenfunction expansion) combined with the results of C. S. Morawetz and D. Ludwig [1968] to obtain $\mathcal{L}^{p}-\mathcal{L}^{q}$ estimates for more general equations with a damping term. Thus he was able to treat the exterior Dirichlet problem.

To solve exterior boundary value problems without damping is, however, more difficult. The Dirichlet problem for the wave equation was treated by Y. Shibata and Y. Tsutsumi [1986 and 1987] assuming smooth and convex (non-trapping) boundaries. They used difficult energy estimates and the results of B. R. Vainberg (comp. [1989]), and also C. S. Morawetz and D. Ludwig [1968] concerning the asymptotics of the resolvent of the linear problem.

The case of inhomogeneous and anisotropic media is also largely open. There are results by O. Liess [1989] in crystal optics.

Largely unsolved is also the question of existence of global weak solutions in $\mathbb{R}^{3}$ and their uniqueness. Little is known about existence and behaviour of shock waves.

2.3. Elastic media with cubic symmetry. The elastic media that were treated so far are isotropic. Thus it is also interesting to look at anisotropic media and I shall finally report on some work I did together with a student of mine, M. Stoth. To make the calculations simple we choose a medium in $\mathbb{R}^{2}$ with cubic symmetry. We start with the linear case and derive $\mathcal{L}^{p}-\mathcal{L}^{q}$ estimates. The linear equations of 
elasticity then read

$$
U_{t t}-\left(\begin{array}{cc}
a \partial_{1}^{2}+b \partial_{2}^{2} & (b+c) \partial_{1} \partial_{2} \\
(b+c) \partial_{1} \partial_{2} & b \partial_{1}^{2}+a \partial_{2}^{2}
\end{array}\right) U=0
$$

Using Fourier transformation this reads

$$
\widehat{U}_{t t}+\left(\begin{array}{cc}
a \xi_{1}^{2}+b \xi_{2}^{2} & (b+c) \xi_{1} \xi_{2} \\
(b+c) \xi_{1} \xi_{2} & b \xi_{1}^{2}+a \xi_{2}^{2}
\end{array}\right) \widehat{U}=0
$$

Let

$$
P(\lambda, \xi):=\lambda \mathrm{id}-\left(\begin{array}{cc}
a \xi_{1}^{2}+b \xi_{2}^{2} & (b+c) \xi_{1} \xi_{2} \\
(b+c) \xi_{1} \xi_{2} & b \xi_{1}^{2}+a \xi_{2}^{2}
\end{array}\right) .
$$

Then $\left\{(\lambda, \xi) \in \mathbb{R}^{3} \mid \operatorname{det} P(\lambda, \xi)=0\right\}$ is the characteric manifold of $P$, and $S:=$ $\left\{\xi \in \mathbb{R}^{2} \mid \operatorname{det} P(1, \xi)=0\right\}$ is called Fresnel's wave surface.

The constants $a, b, c$ are not completely free. From physical considerations $a$, $b$ have to be positive and $|c|<a$. In case $a=2 b+c$ the medium is isotropic. Mathematically simple and interesting are also the cases $c=-b$ (a weakly coupled system), and $a=b$.

We have

$$
\begin{aligned}
\operatorname{det} P(\lambda, \xi) & =\lambda^{2}-\lambda(a+b)|\xi|^{2}+a b\left(\xi_{1}^{4}+\xi_{2}^{4}\right)+\left(a^{2}-2 b c-c^{2}\right) \xi_{1}^{2} \xi_{2}^{2} \\
& =\left(\lambda-\lambda_{1}(\xi)\right)\left(\lambda-\lambda_{2}(\xi)\right) .
\end{aligned}
$$

Thus we obtain two eigenvalues $\lambda_{1}(\xi)$ and $\lambda_{2}(\xi)$. Let $P_{i}(\xi)$ be the projectors on the corresponding eigenspaces and $\mu_{i}(\xi):=\lambda_{i}(\xi)^{1 / 2}$. Assuming $U(0)=: U^{0}=0$ and $U_{t}(0)=: U^{1}$ we then get

$$
\widehat{U}(t)=\frac{\sin \mu_{1} t}{\mu_{1}} P_{1} \widehat{U}^{1}+\frac{\sin \mu_{2} t}{\mu_{2}} P_{2} \widehat{U}^{1} .
$$

We have to estimate, for example,

$$
V(t, x):=\int_{\mathbb{R}^{2}} \mathrm{e}^{\mathrm{i} x \xi} \frac{\sin \mu_{1}(\xi) t}{\mu_{1}(\xi)}\left(P_{1} \widehat{U}^{1}\right)(\xi) d \xi .
$$

In the isotropic case $(a=2 b+c)$ we obtain $\lambda_{1}=a|\xi|^{2}, \lambda_{2}=b|\xi|^{2}$ and

$$
P_{1,2}=\frac{1}{|\xi|^{2}}\left(\begin{array}{cc}
\xi_{1}^{2} & \pm \xi_{1} \xi_{2} \\
\pm \xi_{1} \xi_{2} & \xi_{2}^{2}
\end{array}\right) .
$$

$V$ then has the same structure as the corresponding integral of the wave equation and one therefore obtains the same asymptotics.

In case of $a=b=1, c=0$ we get

$$
\lambda_{1,2}(\xi)=\xi^{2} \pm \xi_{1} \xi_{2} \geq \frac{1}{2} \xi^{2}
$$

and

$$
P_{1,2}=\frac{1}{2}\left(\begin{array}{cc}
1 & \pm 1 \\
\pm 1 & 1
\end{array}\right) .
$$


Choosing

$$
z:=r\left(\begin{array}{c}
\cos \varphi \\
\sin \varphi
\end{array}\right) \quad \text { and } \quad \xi=\xi(z)=\frac{1}{\sqrt{3}}\left(\begin{array}{cc}
\sqrt{3} & 1 \\
-\sqrt{3} & 1
\end{array}\right) z=: A z
$$

we obtain

$$
z=\frac{1}{2}\left(\begin{array}{cc}
1 & -1 \\
\sqrt{3} & \sqrt{3}
\end{array}\right) \xi \quad \text { and } \quad \operatorname{det} \frac{\partial\left(\xi^{1}, \xi^{2}\right)}{\partial(r, \varphi)}=\frac{2 \sqrt{3}}{3} r .
$$

Furthermore, we know $\lambda_{1}(\xi(z))=\left(\xi^{2}+\xi_{1} \xi_{2}\right)(z)=|z|^{2}$. Thus

$$
V(t, x)=\frac{2}{\sqrt{3}} \int_{0}^{\infty} \int_{0}^{2 \pi} \mathrm{e}^{\mathrm{i} x \xi(z)} \frac{\sin |z| t}{|z|} P_{1} \widehat{U}^{1}(\xi(z)) r d \varphi d r,
$$

or with $y:=A^{\operatorname{tr}} x$,

$$
V(t, x)=\frac{2}{\sqrt{3}} \int_{\mathbb{R}^{2}} \mathrm{e}^{\mathrm{i} y z} \frac{\sin |z| t}{|z|} P_{1} \widehat{U}^{1}(A z) d z .
$$

Therefore the solutions asymptotically behave like the solutions of the wave equation. We therefore again get the same asymptotics.

The general case is more interesting. Since we are interested in estimating the energy only, we consider

$$
w(t, x):=\int_{\mathbb{R}^{2}} \mathrm{e}^{\mathrm{i}|\xi|\left\{x \xi_{0}+\mu\left(\xi_{0}\right) t\right\}} h(\xi) d \xi .
$$

Our aim is an estimate of the form

$$
\|w(t)\|_{\mathcal{L}^{\infty}} \leq \frac{c}{(1+t)^{s}}\|h\|_{\mathcal{L}_{, 1}^{\infty}}
$$

Here we assume $h \in \stackrel{\circ}{\mathcal{C}}_{\infty}\left(\mathbb{R}^{2}\right)$, and $c$ may depend on supp $h$. Furthermore,

$$
0=\mu^{4}-(a+b) \mu^{2}+A(\varphi) \quad \text { with } \quad A(\varphi)=f \cos 4 \varphi+g
$$

and

$$
\xi_{0}:=\left(\begin{array}{c}
\cos \varphi \\
\sin \varphi
\end{array}\right), \quad f:=\frac{2 a b-d}{8}, \quad g:=\frac{6 a b+d}{8}, \quad d:=a^{2}+b^{2}-(b+c)^{2} .
$$

Let $r:=1 / \mu$, let $k$ be the curvature of

$$
r(\varphi) \cdot\left(\begin{array}{c}
\cos \varphi \\
\sin \varphi
\end{array}\right)
$$

and $n(\varphi)$ its normal. We can attach to each $x$ a "point of stationary phase" $\varphi_{x}$. Then $n\left(\varphi_{x}\right)$ has the same direction as $x$ and using stationary phase methods we obtain

$$
w(t, x)=\mathcal{O}\left(1 /(1+t)^{1 / 2}\right)
$$


if $k\left(\varphi_{x}\right) \neq 0$. Furthermore,

$$
\begin{array}{ll}
w(t, x)=\mathcal{O}\left(1 /(1+t)^{1 / 3}\right) & \text { if } k\left(\varphi_{x}\right)=0 \text { and } k^{\prime}\left(\varphi_{x}\right) \neq 0, \\
w(t, x)=\mathcal{O}\left(1 /(1+t)^{1 / 4}\right) & \text { if } k\left(\varphi_{x}\right)=0 \text { and } k^{\prime}\left(\varphi_{x}\right)=0 .
\end{array}
$$

The first case corresponds to normal behaviour in $\mathbb{R}^{2}$, as found with the wave equation or isotropic elastic medium. But the two other cases can also be found. Choosing $a=5$ and $b=2$ we get

$$
\begin{array}{ll}
k=0, k^{\prime} \neq 0 & \text { for } c=4.9 \text { and } \varphi=0.471, \\
k=0, k^{\prime}=0 & \text { for } c=0 \text { and } \varphi=\pi / 4, \\
k=0, k^{\prime}=0 & \text { for } c=\sqrt{15}-2=1.873 \text { and } \varphi=0 .
\end{array}
$$

To illustrate this, the Fresnel surfaces for different coefficients are shown in Figures $1-6$;

is plotted there.

$$
\varphi \rightarrow r(\varphi) \cdot\left(\begin{array}{c}
\cos \varphi \\
\sin \varphi
\end{array}\right)
$$
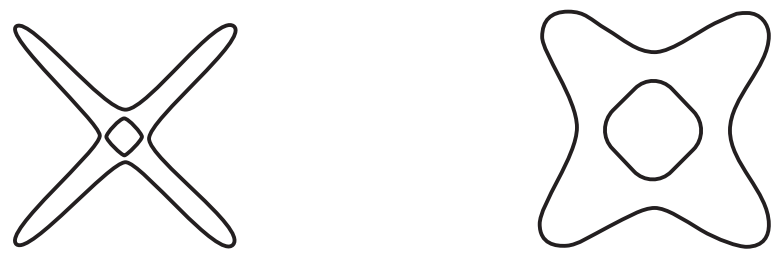

Fig. 1. Left: $a=5, b=2, c=4$.9. Right: $a=5, b=2, c=4$
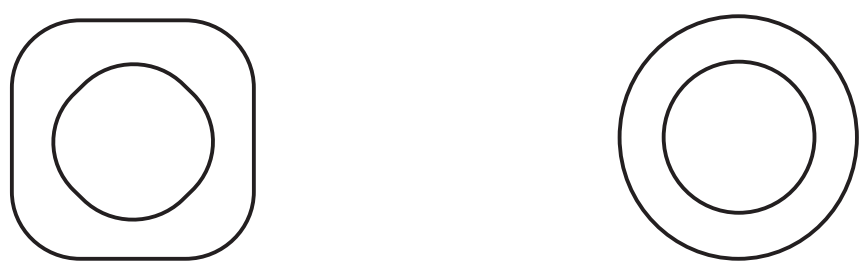

Fig. 2. Left: $a=5, b=2, c=1.87$. Right: $a=5, b=2, c=1$
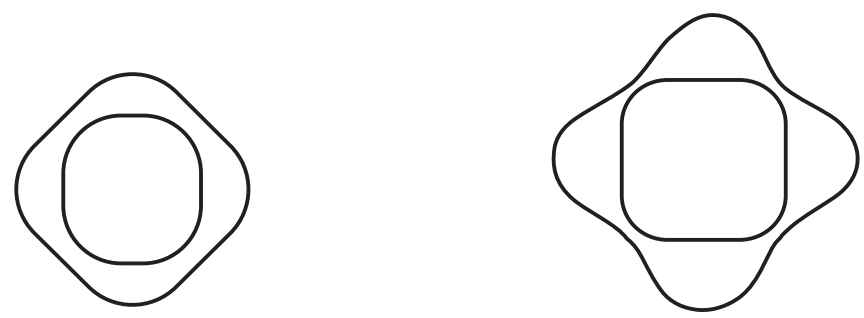

Fig. 3. Left: $a=5, b=2, c=0$. Right: $a=5, b=2, c=-1.5$ 


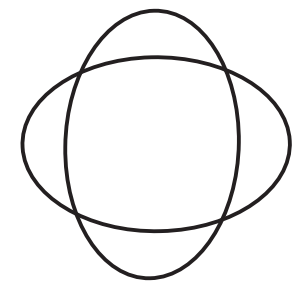

Fig. 4. $a=5, b=2, c=-2$
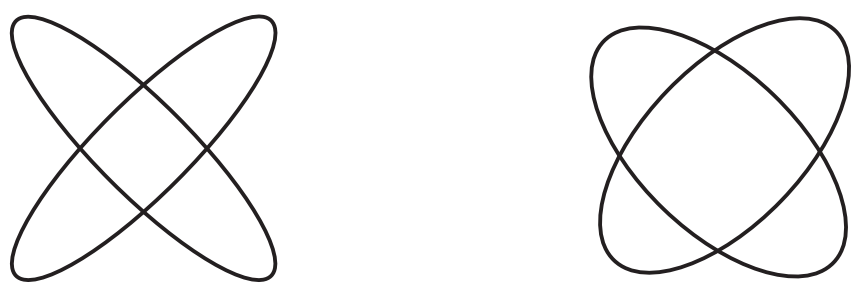

Fig. 5. Left: $a=1, b=1, c=0.75$. Right: $a=1, b=1, c=0$
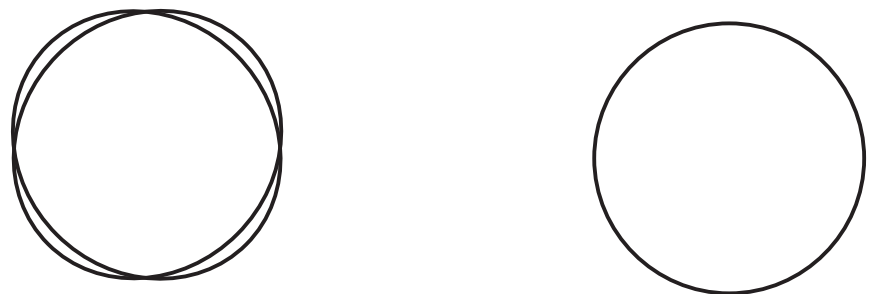

Fig. 6. Left: $a=1, b=1, c=-0.75$. Right: $a=1, b=1, c=-1$

Let us indicate the proof. On setting

$\zeta=\mu \xi, \quad x=|x|\left(\begin{array}{c}\cos \psi \\ \sin \psi\end{array}\right), \quad g(\zeta)=\frac{1}{\mu^{2}} h\left(\frac{\zeta}{\mu}\right), \quad f(t, \varphi)=\frac{|x| \cos (\psi-\varphi)}{\mu(\varphi)}+t$,

we obtain

$$
w(t, x)=\int_{\mathbb{R}^{2}} \mathrm{e}^{\mathrm{i}|\zeta| f(t, \varphi)} g(\zeta) d \zeta .
$$

For $g \in \stackrel{\circ}{\mathcal{C}}_{\infty}\left(\mathbb{R}^{2}\right)$ and with constants $\gamma$ depending on $\operatorname{supp} g$ this can be estimated as follows.

$t$ small: For $t<1$ we obtain $|w(t, x)| \leq \gamma\|g\|_{\mathcal{L}^{\infty}} \leq \gamma\|h\|_{\mathcal{L}^{\infty}}$.

$t$ large: Assume $t \geq 1$. Depending on $a, b$ and $c$ positive constants $p, q$ exist with $p \leq r(\varphi) \leq q$. In case $t \geq 2 q|x|$ we then get

$$
f \geq-|x| r+t \geq-|x| q+t \geq t / 2
$$


and thus by partial integration

$$
|w(t, x)| \leq \frac{\gamma}{t}\|h\|_{\mathcal{L}_{, 1}^{\infty}} .
$$

The case $t<2 q|x|$ is more interesting. Starting from

$$
f^{\prime}(\varphi):=\partial_{\varphi} f(t, \varphi)=\frac{|x|}{\mu^{2}(\varphi)}\left\{\mu(\varphi) \sin (\psi-\varphi)-\mu^{\prime}(\varphi) \cos (\psi-\varphi)\right\}
$$

we define a point of stationary phase $\tau$ by $f^{\prime}(\tau)=0$ or $\tan (\psi-\varphi)=\mu^{\prime} / \mu(\varphi)$. If $\varphi \notin[\tau-\delta, \tau+\delta]$ we obtain from $\left|f^{\prime}(\varphi)\right| \geq \gamma|x|$

$$
|w(t, x)| \leq \frac{\gamma}{|x|}\|h\|_{\mathcal{L}^{\infty}} \leq \frac{\gamma}{t}\|h\|_{\mathcal{L}^{\infty}} .
$$

What remains is $\varphi \in(\tau-\delta, \tau+\delta)$ and the behaviour of $w(t, x)$ depends on whether

$$
f^{\prime \prime}(\tau) \neq 0,
$$

or

$$
f^{\prime \prime}(\tau)=0 \quad \text { and } \quad f^{\prime \prime \prime}(\tau) \neq 0
$$

or

$$
f^{\prime \prime}(\tau)=f^{\prime \prime \prime}(\tau)=0 \quad \text { and } \quad f^{\mathrm{iv}}(\tau) \neq 0 .
$$

$f^{\prime \prime}(\tau)=0$ is equivalent to $\left(\mu+\mu^{\prime \prime}\right)(\tau)=k(\tau)=0$. Thus we are finally led to three possible cases which, using the stationary phase method, yield the desired estimates of $w$. Further details can be found in Stoth [1991].

Depending on the coefficients one thus obtains the decay coefficients

$$
s_{1}=1 / 2, \quad s_{2}=1 / 3, \quad s_{3}=1 / 4 .
$$

For the wave equation in $\mathbb{R}^{2}$ one knows $s=1 / 2$.

Thus the $\mathcal{L}^{p}-\mathcal{L}^{q}$ estimate (2.3) also gets worse for cubic elastic media. From (2.8) and a resultat by Pecher [1976] we obtain with $u:=D U$ in $\mathbb{R}^{2}$

$$
\|u(t)\|_{\mathcal{L}^{q}} \leq c_{q}(1+t)^{-s(1-2 / q)}\left\|u^{0}\right\|_{\mathcal{L}_{, N_{p}}^{p}}
$$

for $1<p \leq 2,1 / p+1 / q=1$ with $2(2-p) / p \leq N_{p}<2(2-p) / p+1$.

We can thus prove the existence of global smooth solutions of the quasilinear system of elasticity

$$
\partial_{t}^{2} U_{i}=C_{i m j n}(\nabla U) \partial_{m} \partial_{n} U_{j}
$$

with initially cubic symmetry only under a stronger restriction on the nonlinearity. If

$$
\left|C_{i m j n}(\nabla U)-C_{i m j n}(0)\right| \leq c|\nabla U|^{\alpha-1}
$$

then such solutions exist as indicated in the following table:

\begin{tabular}{c||c|c|c}
\hline$s$ & $\alpha$ & $p$ & $q$ \\
\hline $1 / 2$ & 4 & $8 / 7$ & 8 \\
$1 / 3$ & 5 & $10 / 9$ & 10 \\
$1 / 4$ & 6 & $12 / 11$ & 12 \\
\hline
\end{tabular}


Aluminium, copper and nickel are anisotropic cubic media. For aluminium we find the decay coefficient $1 / 2$, and for copper and nickel $1 / 3$ :

\begin{tabular}{l||c|c|c|c}
\hline & $a$ & $b$ & $c$ & $s$ \\
\hline Aluminium & 9.5 & 2.8 & 4.9 & $1 / 2$ \\
Copper & 25.2 & 12.2 & 15.4 & $1 / 3$ \\
Nickel & 17 & 7.55 & 12.3 & $1 / 3$ \\
\hline
\end{tabular}

\section{References}

H. D. Alber and R. Leis [1988], Initial-boundary value and scattering problems in mathematical physics, in: Lecture Notes in Math. 1357, Springer, 23-60.

A. L. Belopol'skiu and M. Sh. Birman [1968], The existence of wave operators in the theory of scattering with a pair of spaces, Math. USSR-Izv. 2, 1117-1130.

J. Bergh and J. Löfström [1976], Interpolation Spaces. An Introduction, Springer, Berlin.

D. Christodoulou [1986], Global solutions of nonlinear hyperbolic equations for small data, Comm. Pure Appl. Math. 39, 267-287.

G. F. D. Duff [1960], The Cauchy problem for elastic waves in an anisotropic medium, Philos. Trans. Roy. Soc. London Ser. A 252, 249-273.

D. M. Èı̆dus [1962], The principle of limiting absorption, Mat. Sb. 57 (99), 13-44 and AMS Transl. (2) 47 (1965), 157-191.

A. Erdélyi [1956], Asymptotic Expansions, Dover, New York.

H. Freudenthal [1939], Über ein Beugungsproblem aus der elektromagnetischen Lichttheorie, Compositio Math. 6, 221-227.

L. Hörmander [1976], The boundary problems of physical geodesy, Arch. Rational Mech. Anal. $62,1-52$.

T. Ikebe [1960], Eigenfunction expansions associated with the Schrödinger operators and their applications to scattering theory, ibid. 5, 1-34.

S. Jiang [1988], Global existence and asymptotic behaviour of smooth solutions in one-dimensional nonlinear thermoelasticity, thesis, University of Bonn.

S. Jiang [1990a], Far field behavior of solutions to the equations of nonlinear 1-d-thermoelasticity, Appl. Anal. 36, 25-35.

S. Jiang [1990b], Numerical solution for the Cauchy Problem in nonlinear 1-d-thermoelasticity, Computing 44, 147-158.

S. Jiang and R. Racke [1990], On some quasilinear hyperbolic-parabolic initial boundary value problems, Math. Methods Appl. Sci. 12, 315-339.

F. John [1974], Formation of singularities in one-dimensional nonlinear wave propagation, Comm. Pure Appl. Math. 27, 377-405.

F. John [1976], Delayed singularity formation in solutions of nonlinear wave equations in higher dimensions, ibid. 29, 649-681.

F. John [1977], Finite amplitude waves in a homogeneous isotropic elastic solid, ibid. 30, 421446.

F. John [1981], Blow-up for quasi-linear wave equations in three space dimensions, ibid. 34, $29-51$.

F. John [1983], Formation of singularities in elastic waves, in: Trends and Applications of Pure Mathematics to Mechanics, Proceedings Palaiseau 1983, P. G. Ciarlet and M. Roseau (eds.), Lecture Notes in Phys. 195, Springer, 194-210. 
F. John [1986a], Long time effects of nonlinearity in second order hyperbolic equations, Comm. Pure Appl. Math. 39 (S), 139-148.

F. John [1986b], Partial Differential Equations, Appl. Math. Sci. 1, Springer, New York.

F. John [1987], Existence for large times of strict solutions of nonlinear wave equations in three space dimensions for small initial data, Comm. Pure Appl. Math. 40, 79-109.

F. John [1988], Almost global existence of elastic waves of finite amplitude arising from small initial disturbances, ibid. 41, 615-666.

F. John [1990], Nonlinear Wave Equations, Formation of Singularities, Univ. Lecture Ser. No 2, Amer. Math. Soc. Providence, R.I.

K. Jörgens [1961], Das Anfangswertproblem im Großen für eine Klasse nichtlinearer Wellengleichungen, Math. Z. 77, 295-308.

T. Kato [1970], Linear evolution equations of "hyperbolic" type, J. Fac. Sci. Univ. Tokyo 17, $241-258$.

T. Kato [1973], Linear evolution equations of "hyperbolic" type, II, J. Math. Soc. Japan 25, 648-666.

T. Kato [1975a], Quasi-linear equations of evolution, with applications to partial differential equations, in: Lecture Notes in Math. 448, Springer, 25-70.

T. Kato [1975b], The Cauchy problem for quasi-linear symmetric hyperbolic systems, Arch. Rational Mech. Anal. 58, 181-205.

T. Kato [1976], Perturbation Theory for Linear Operators, Springer, Berlin.

T. Kato [1985], Abstract Differential Equations and Nonlinear Mixed Problems, Fermi Lectures. Scuola Norm. Sup. Pisa.

S. Kawashima [1983], Systems of a hyperbolic-parabolic composite type, with applications to the equations of magnetohydrodynamics, thesis, Kyoto University.

S. Klain erman [1980], Global existence for nonlinear wave equations, Comm. Pure Appl. Math. 33, 43-101.

S. Klainerman [1982], Long-time behavior of solutions to nonlinear evolution equations, Arch. Rational Mech. Anal. 78, 73-98.

S. Klainerman [1985], Uniform decay estimates and the Lorentz invariance of the classical wave equation, Comm. Pure Appl. Math. 38, 321-332.

S. Klainerman [1986], The null condition and global existence to nonlinear wave equations, Lectures in Appl. Math. 23, Amer. Math. Soc., 293-326.

S. Klainerman and G. Ponce [1983], Global, small amplitude solutions to nonlinear evolution equations, Comm. Pure Appl. Math. 36, 133-141.

V. D. Kupradze [1934], Über das „Ausstrahlungsprinzip“ von A. Sommerfeld, Dokl. Akad. Nauk SSSR 1, 55-58.

P. D. Lax and R. S. Phillips [1989], Scattering Theory, Academic Press, Boston.

R. Leis [1980], Außenraumaufgaben in der linearen Elastizitätstheorie, Math. Methods Appl. Sci. 2, 379-396.

R. Leis [1981], Über das asymptotische Verhalten thermoelastischer Wellen im $\mathbb{R}^{3}$, ibid. 3, 312317.

R. Leis [1986], Initial Boundary Value Problems in Mathematical Physics, Wiley and B. G. Teubner, Stuttgart.

R. Leis [1989], Initial-boundary value problems in elasticity, in: Pitman Res. Notes Math. 216, 73-96.

O. Liess [1989], Global existence for the nonlinear equations of crystal optics, Journ. "Équ. aux Dér. Par.", Exp. No. V, 11 pp., École Polyt., Palaiseau.

C. S. Morawetz [1975], Decay for solutions of the exterior problem for the wave equation, Comm. Pure Appl. Math. 28, 229-264.

C. S. Morawetz and D. Ludwig [1968], An inequality for the reduced wave operator and the justification of geometrical optics, Comm. Pure Appl. Math. 21, 187-203. 
C. S. Morawetz, W. Ralston and W. Strauss [1977], Decay of solutions of the wave equation outside nontrapping obstacles, ibid. 30, 447-508 and 31, 795.

J. Moser [1961], A new technique for the construction of solutions of nonlinear differential equations, Proc. Nat. Acad. Sci. U.S.A. 47, 1824-1831.

C. Müller [1952], Zur Methode der Strahlungskapazität von H. Weyl, Math. Z. 56, 80-83.

C. Müller [1954], On the behavior of the solutions of the differential equation $\Delta u=F(x, u)$ in the neighborhood of a point, Comm. Pure Appl. Math. 7, 505-515.

D. B. Pearson [1978], A generalization of the Birman trace theorem, J. Funct. Anal. 28, 182186.

H. Pecher [1976], $\mathcal{L}^{p}$-Abschätzungen und klassische Lösungen für nichtlineare Wellengleichungen I, Math. Z. 150, 159-183.

R. Racke [1988], Initial boundary value problems in thermoelasticity, in: Partial Differential Equations and Calculus of Variations, Lecture Notes in Math. 1357, Springer, 341-358.

$\mathrm{R}$. Racke [1990a], $\mathcal{L}^{p}-\mathcal{L}^{q}$ estimates for solutions to the equations of linear thermoelasticity in exterior domains, Asymptotic Anal. 3, 105-132.

R. Racke [1990b], A unique continuation principle and weak asymptotic behaviour of solutions to semilinear wave equations in exterior domains, Appl. Math. Letters 3, 53-56.

R. Racke [1990c], Blow-up in nonlinear three-dimensional thermoelasticity, Math. Methods Appl. Sci. 12, 267-273.

R. Racke [1990d], Decay rates for solutions of damped systems and generalized Fourier transforms, J. Reine Angew. Math. 412, 1-19.

R. Racke [1990e], On the Cauchy problem in nonlinear 3-d-thermoelasticity, Math. Z. 203, 649-682.

R. Racke and G. Ponce [1990], Global existence of solutions to the initial value problem for nonlinear thermoelasticity, J. Differential Equations 87, 70-83.

R. Racke and S. Zheng [1991], Global existence of solutions to a fully nonlinear fourth-order parabolic equation in exterior domains, Nonlinear Anal., to appear.

M. Reed and B. Simon [1972-79], Methods of Modern Mathematical Physics I - IV, Academic Press, New York.

F. Rellich [1943], Über das asymptotische Verhalten der Lösungen von $\Delta u+\lambda u=0$ in unendlichen Gebieten, Jber. Deutsch. Math.-Verein. 53, 57-65.

J. Schauder [1935], Das Anfangswertproblem einer quasilinearen hyperbolischen Differentialgleichung zweiter Ordnung in beliebiger Anzahl von unabhängigen Veränderlichen, Fund. Math. 24, 213-246.

Y. Shibata and Y. Tsutsumi [1986], On a global existence theorem of small amplitude solutions for nonlinear wave equations in an exterior domain, Math. Z. 191, 165-199.

Y. Shibata and Y. Tsutsumi [1987], Local existence of solution for the initial boundary value problem of fully nonlinear wave equation, Nonlinear Anal. 11, 335-365.

M. Stoth [1991], Globale klassische Lösungen der quasilinearen Elastizitätsgleichungen für kubisch elastische Medien im $\mathbb{R}^{2}$, SFB 256 - Preprint \#157, Universität Bonn.

W. A. Strauss [1989], Nonlinear Wave Equations, CBMS Regional Conf. Ser. in Math. 73, Amer. Math. Soc., Providence, R.I.

B. R. Vainberg [1989], Asymptotic Methods in Equations of Mathematical Physics, Gordon and Breach, New York.

I. N. Vekua [1967], New Methods for Solving Elliptic Equations, North-Holland, Amsterdam.

M. Vishik and O. A. Ladyzhenskaya [1956], Boundary value problems for partial differential equations and certain classes of operator equations, Uspekhi Mat. Nauk 11 (6,72), 41-97 and AMS Transl. (2) 10, 223-281.

H. Weyl [1952], Kapazität von Strahlungsfeldern, Math. Z. 55, 187-198.

C. H. Wilcox [1975], Scattering Theory for the d'Alembert Equation in Exterior Domains, Lecture Notes in Math. 442, Springer, Berlin. 